\title{
The Office Vaginoscopic Hysteroscopy in Management of Virginal Women with Recurrent Abnormal Uterine Bleeding
}

\author{
Yi Liang Lee ${ }^{1}$, Yin Shiuan Bai ${ }^{2}$ and Chang Sheng Yin ${ }^{2 *}$ \\ ${ }^{1}$ Department of Obstetrics and Gynecology, Cathay General Hospital, Taiwan \\ ${ }^{2}$ Department of Obstetrics and Gynecology, Kang Ning Hospital, Taiwan
}

*Corresponding author: Chang Sheng Yin, Department of Obstetrics and Gynecology, Kang Ning Hospital, no.26, lane 420, sec 5, Cheng Kung Rd, Neihu, Taipei, Taiwan.

Received Date: February 26, 2019

Published Date: March 01, 2019

\section{Abstract}

Introduction: Abnormal uterine bleeding (AUB) is the most common reason to visit gynecology outpatient clinic. Virginal women are a challenge for gynecologist in management of AUB. The office vaginoscopic hysteroscopy is an ideal selection. The usefulness and feasibility of no touch hysteroscopy in virginal women is rarely reported. AUB.

Objectives: The feasibility of vaginoscopic hysteroscopy for management of virginal women with abnormal uterine bleeding

Materials and methods: Patients: A prospective observational study of 36 virginal women with AUB seen at the Kang Ning Hospital during a 10 months period, from 1st Jan to 31st Oct 2018 was included.

Methods: Rigid hysteroscopy, $4 \mathrm{~mm}$ out diameter (OD) was used after the initial clinical evaluation and transabdominal sonography.

Result: A total of 36 consecutive women, aged 16 to 52 (mean 31) with AUB was included. The hysteroscopy was successful in 31 out of $36(86 \%)$, while incomplete view $4(11 \%)$. One failed examination (3\%) was excluded. A total of 35 women were included for study. Thirteen endometrial polyps (37\%) and 2 submucosal leiomyomas (6\%) were found. Total operation time: mean time 3 $\mathrm{m} 29 \mathrm{~s} \pm 1 \mathrm{~m} 16 \mathrm{~s}$ (range $1 \mathrm{~m} 54 \mathrm{~s}-11 \mathrm{~m} 31 \mathrm{~s}$ ). Patients rated pain score: $3 \pm 1.6$ (range 1-8). There was no hymeneal injury, only one vasovagal attack occurred. Thirteen women underwent see and treat endometrial biopsy no malignancy was found. Subsequent two submucosal myomectomy with hymenoplasty were performed.

Conclusion: The office vaginoscopic hysteroscopy is safe and feasible for assessment of virginal women with AUB.

Keywords: Virginal women; Vaginoscopic hysteroscopy; Abnormal uterine bleeding; Endometrial polyp; Submucosal leiomyomas; Hymenoplasty.

\section{Introduction}

Abnormal uterine bleeding (AUB) is defined as any abnormality in menstrual bleeding of quantity, duration, or schedule. The normal menstrual cycle is typically between 24 days and 38 days and lasts up to 8 days. AUB account for up to $20 \%$ of all gynecologic visits. The etiology of AUB is broad. In 2010, the PALM-COEIN classification system for AUB, has been approved by the FIGO [1]. The most common causes in nonpregnant women are endocrine dysfunction, then benign tumors, neoplasia, others. The initial evaluation include history, physical examination, laboratory tests, further evaluation included pelvic ultrasound, endometrial biopsy, and or hysteroscopy. Virginity, a personal philosophy, is a sensitive issue and challenge for gynecologist in management of AUB. The vaginoscopic hysteroscopy had been introduced for diagnosis and treatment in women with intact hymen. A systematic review in 2015 identified 19 case reports limited to diagnosis and therapeutic usage in pediatric gynecology [2]. There are only two 
case series reports in adult women $[3,4]$. In this study we assessed the usefulness and feasibility of vaginoscopic hysteroscopy for management of virginal women with AUB.

\section{Materials and Methods}

\section{Patients}

A prospective observational study of 36 women, aged 16 to 53, who declared virginity with abnormal AUB who agree to examination at the Kang Ning Hospital during a 10 months period, from 1st Jan to 31st Oct 2018 was included.

\section{Exclusion criteria included}

Bleeding from cervix and vagina, history of bleeding dyscrasias, use of anticoagulant medications, use of hormonal replacement therapy or oral contraceptive pills, clinical examination showing no other pelvis organ pathology.

\section{Methods}

The vaginoscopic rigid hysteroscopy was performed after the initial clinical evaluation and transabdominal ultrasound examination. All procedures were usually performed in proliferative phase using a $4 \mathrm{~mm}$ continuous flow hysteroscope. The detailed procedure of the vaginoscopic hysteroscopy had been reported previously [5]. No preoperative medications or routine prophylactic antibiotics was given. The result of hysteroscopic procedure was defined as complete (clear view of cavity and both tubal ostia, cervical canal, cervix), incomplete (part of cavity obscured), and failed examination (pain, or difficult entry). At the end of the examination in thirteen women, the hysteroscope tip was agitated within the endometrium in order to obtain a tissue sample from manual vacuum aspirator (MVA). Endometrial biopsy was performed using a sharpened tip of $4 \mathrm{~mm}$ flexible Karman cannula which is inserted into the uterus through the hysteroscopy out sheath after removal of telescope. Immediately after each procedure patients rated the visual analog scale scores (range 0-10).

All authors followed The Declaration of Helsinki: ethical principles for medical research involving human subjects. The hospital ethics committee approved this study (KNH107-11). All patients who undergoing these procedures had a signed informed consent. All data was analyzed using the Microsoft Excel software. Mean \pm SD were calculated for age, BMI, operation time, and percentage was calculated for distribution of age, BMI, correlation between hysteroscopic finding.

\section{Result}

A total of 36 consecutive women, aged 16 to 52 (mean $31 \pm 10$ ) with AUB was included. The hysteroscopy was successful in 31 out of 36 (86\%), incomplete view in 4(11\%). One failed examination (3\%) was excluded for analysis. Nine women underwent concomitant endometrial biopsy and no malignancy was found. The detailed of the 35 women were included for study.

Total operation time: mean 3 minutes 29 second \pm 1 minute 16 seconds range 1 minute 54 seconds- 11 minutes 31 seconds. Patients rated pain score: $3 \pm 1$.6 (range 1-8)
Thirteen endometrial polyps (37\%) and 2 submucosal leiomyomas $(9 \%)$ were found. There were no vulva and vaginal injury, but one vasovagal attack occurred during hysteroscopic examination and with endometrial biopsy. There is no other complication. Two women (43, 34-year-old) underwent hysteroscopic myomectomy at another session and hymenorrhaphy were done after surgery.

\section{Discussion}

The gynecological examination in women with intact hymen is usually incomplete. AUB a common and alarming symptom, the differential diagnosis is broad. Pelvic ultrasound is usually the initial modality for imaging uterine pathology in women with AUB. Two basic gynecologic ultrasound imaging scanning approaches are: transabdominal and transvaginal. Transabdominal sonography is difficult to evaluate the uterus with retroverted or retroflexed position, but still is the mainstay of endometrial evaluation in women with intact hymen. Several historically instruments had been tried, such as a modified Foley catheter by Redman, a balloon vaginoscopic by Terruhn, in 1990 Major used a No.26 Fr. Resectoscope with continuous fluid irrigation as vaginoscopic approach [5]. The usefulness on vaginoscopic approach for diagnosis and treatment had been reported in adolescent girls by a systematic review by Johary el al. [2]. Current vaginoscopic minihysteroscopy (less than $5 \mathrm{~mm}$ ) is an ideal tool for management of AUB in virginal women. The $4 \mathrm{~mm}$ hysteroscope we used in this study is the same diameter of internal cervical os. Previous two case series studies by Xu D and Küçük T reported a total of 40 women with heterogenous indications while only 21 of them was AUB $[3,4]$. To the best of our knowledge, our case series is the largest one ever reported. In this study, our successful rate was $97 \%$, only one failed. It is comparable to previous 2 case series. The resistant cervix, in fact, distorted cervical canal, and angulation between uterine corpus and cervix, is the most common causes of failure. Some difficulties may encounter in anteflexed or retroflexed uterus that have to be overcome. Xu advised pressure applied above the symphysis if the uterus is anteflexed, and retroflexed put fingers in patient' rectum to apply pressure to move the uterus to a mid-position [3]. Küçük $\mathrm{T}$ recommended the obstetric McRoberts maneuver to downward placement of the uterus [4]. The best position of the uterus for hysteroscopy is mid-position [6]. There are still several obstacles: patients' comprehension, potential hymeneal injury, and image quality.

In this study we found endometrial polyps were most frequent structural causes of AUB in virginal women following by submucosal leiomyomas. In our study 13 women permitted undergoing the see and endometrial biopsy, normal endometrium was found. In our report two submucosal myomectomy with concomitantly hymenoplasty were performed. There is a concern about hymenoplasty after a torn hymen during hysteroscopic myomectomy. While in virginal women with management of AUB, a potential hymeneal injury needs to be discussed. Virginity is a personal philosophy.

The virginity rate is differing greatly between Eastern and Western society. In Eastern society like Taiwan, there is a lot 
of pressure and a social taboo on women to lose their virginity before marriage, must be respected by medical professions. After counseling then we performed the hymenoplasty. The prevalence of AUB according to FIGO classification in Chinese women by Sun et al showed the ovulatory dysfunction (AUB-O) is the most common, followed endometrial polyps (AUB-P), uterine fibroids (AUB-L) [7]. Our study showed similar finding as Sun's. Previous reports limits to case reports and case series, larger studies are needed. In our preliminary experience the correlation between hysteroscopy and intrauterine pathology is the same as an experience of 10,500 diagnostic hysteroscopes by Cicinelli et al, a very high diagnostic accuracy for normal endometrium, atrophy, myomas, polyps, uterine malformation, adhesions, and even cancer [8].

\section{Conclusion}

The Vaginoscopic hysteroscopy is an ideal instrument in diagnosis and treatment in virginal women with AUB. This nontraumatic approach protects the state of virginity.

\section{Acknowledgement}

The authors would like to thank Miss Feng-Wei Bai, Pei-Ying Yang RN, Miss LI-Chuan Yao and for their hard work and support throughout this study.

\section{Conflict of Interest}

The authors of this article have no conflict of interest to disclose.

\section{References}

1. Munro MG, Critchley HO, Broder MS, Fraser IS (2011) FIGO classification system (PALM-COEIN) for causes of abnormal uterine bleeding in nongravid women of reproductive age. Int J Gynaecol Obstet 113(1): 3-13.

2. Johary J, Xue M, Xu B, Xu D, Aili A (2015) Use of hysteroscope for vaginoscopy or hysteroscopy in adolescents for the diagnosis and therapeutic management of gynecologic disorders: a systematic review. J Pediatr Adolesc Gynecol 28(1): 29-37.

3. Xu D, Xue M, Cheng C, Wan Y (2006) Hysteroscopy for the diagnosis and treatment of pathologic changes in the uterine cavity in women with an intact hymen. J Minim Invasive Gynecol 13(3): 222-224.

4. Küçük T (2007) When virginity does matter: Rigid hysteroscopy for diagnostic and operative vaginoscopy-A series of 26 cases. J Minim Invasive Gynecol 14(5): 651-653.

5. Major T, Bacskó G, Lampé L, Borsos A (1995) Hysteroscopic vaginoscopy in the diagnosis of vaginal bleeding. Surg Technol Int IV: 220-222.

6. Boschert S (2003) Wider use of office flexible hysteroscopy urged. OB/ GYN News.

7. Sun Y, Wang Y, Mao L, Wen J, Bai W (2018) Prevalence of abnormal uterine bleeding according to new International Federation of Gynecology and Obstetrics classification in Chinese women of reproductive age. A crosssectional study. Medicine (Baltimore) 97(31): e11457.

8. Cicinelli E (2005) Diagnostic minihysteroscopy with vaginoscopic approach: rationale and advantages. J Minim Invasive Gynecol 12(5): 396-400. 